\title{
Ninja Telemetry AMT Survival in a Congested Spectral Environment
}

\author{
Timothy A. Chalfant \\ COLSA Corporation, 41240 12th St. West, Palmdale, CA USA \\ tchalfant@colsa.com
}

\begin{abstract}
:
Spectrum encroachment affects Aeronautical Mobile Telemetry (AMT) in many bands and many regions throughout the world. AMT users have found themselves with less spectrum in which to operate, and in some instances, spectrum once allocated exclusively for telemetry, has been opened to sharing with other users. This paper establishes a need for better tools and methodologies to accommodate AMT operations in this "unfriendly sky" when the AMT operator must operate in bands where they may lack a primary allocation (International Telecommunication Union (ITU) and/or domestic), and its associated protection. Of course, any operations on such a (secondary) basis would be for non-safety-of-flight and other non-high-value missions.

In these cases, we must function with the stealth of the ancient Ninjas to avoid causing interference while in turn avoiding interference from primary users in the band. This paper, directed to the Range or Facility executive management, presents a new philosophy and call to adapt to this new paradigm. The telemetry community needs to aggressively research and develop new technology to remain effective in shared spectral environments.
\end{abstract}

Key words: Aeronautical Mobile Telemetry, Radio Frequency (RF) Spectrum, Spectrum Encroachment, Spectrum Efficiency, Quality of Service, Spectrum Sharing

\section{Early Telemetry}

"Those were the days my friend, we thought they'd never end..."

\section{- $\quad$ Song "Those Were the Days" [1]}

Aeronautical Mobile Telemetry (AMT) has a long history in aerospace. Early aerospace telemetry dates back to its space inauguration with the Soviet satellite Sputnik, launched in 1957. The famous "beeping" of Sputnik-1 was in reality a Pulse Duration Modulated telemetry signal, encoding internal pressure (pulse length) and temperature (length between pulses) in the signal.

Telemetry systems advanced rapidly but were limited in their utilization by the technology of the day (large, heavy, lots of power). Early telemetry's unreliability was such that Wernher von Braun once claimed that watching the rocket through binoculars was a better idea [2].
At this time the technology, not radio frequency (RF) spectrum, was the limiting factor.

In the United States (US) early telemetry system were assigned to an allocation in the 2meter band at 225-260 MHz (sometimes referred to as " $P$ " band). A government directive issued in 1958 required telemetry users to vacate this band and move to the 1435-1535 $\mathrm{MHz}$ and 2200-2300 MHz bands ( $\mathrm{L}$ and $\mathrm{S}$ band) by 1970 . We lost $35 \mathrm{MHz}$ but gained 200MHz. Life was good.

Telemetry systems grew to accommodate the increasing need for real-time data. The bands accommodated that growth very well through the 1960's and 70's. The available spectrum was able to accommodate the growing utilization. The slow advances in telemetry technology only limited telemetry growth at that time. 


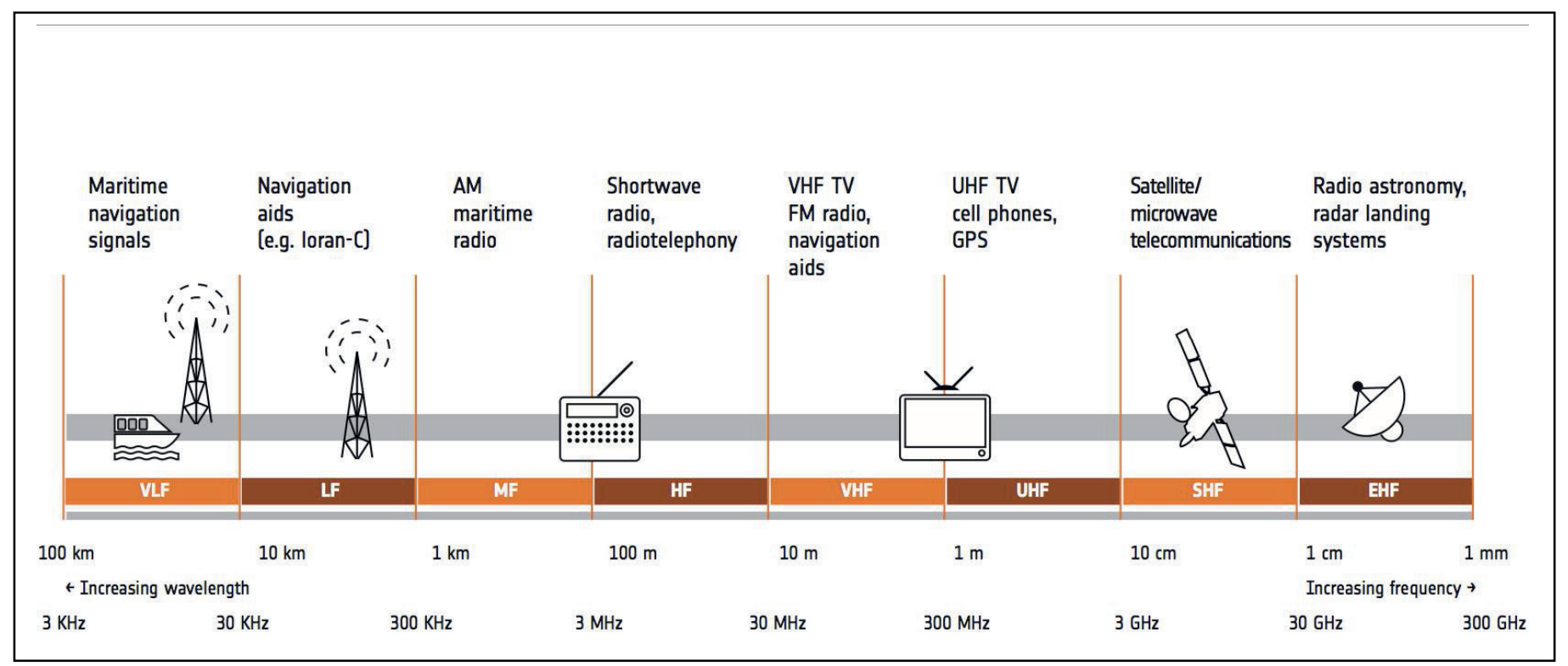

Fig. 1. RF Spectrum

US telemetry bands, managed and controlled by the US Government, were shared with the aerospace industry. Scheduling was done cooperatively between the Government and the aerospace industry (via the Aerospace and Flight Test Radio Coordinating Council [AFTRCC]) [3]. Scheduling was simple with projects being de-conflicted by assignment of dedicated center frequencies, bandwidths, and guard-bands. There were enough frequency assignments to accommodate all users. Once you had your assignment, you could use it whenever you needed.

At that time, flight test telemetry was protected from interference by the frequency coordination with AFTRCC and the government's frequency assignment system. The safety-of-flight needs associated with aerospace vehicles require protection to provide an interference-free environment, as do other high-value missions. AMT is very susceptible to interference. Due to the small size and power restrictions of aeronautical test articles, flight test telemetry is a low-power signal not very far above the noise floor. This meant that large antennas and clean spectrum was needed to find the telemetry "needle in the haystack". These signals were well protected from co-channel and adjacent channel interference by restricted access to these bands per US government's Federal Communications Commission (FCC) and the National Telecommunications and Information Administration (NTIA) regulations and the intelligent scheduling of center frequencies between guard-bands.

Then several things changed....

\section{Growth of Wireless Communication}

The growth of the internet, social media, and wireless communications created a new need for RF spectrum. Over the past 20 years, the world has seen the birth and adolescence of a rapidly growing commercial mobile service industry that has an insatiable appetite for spectrum. This growth has only accelerated. By the end of 2010, there were 5.3 billion mobile cellular subscriptions globally, including 870 million active mobile broadband subscriptions [4].

This has resulted in many national administrations taking a new look at RF spectrum. Increasingly, both industry and governments are viewing spectrum in economic terms, as an input to the production of telecommunications services and a source of revenue.

RF spectrum, once only of interest to scientists and engineers, has become the means to allow users to socially connect and download music and movies. The connected-generation has taken root globally and spectrum is now a limited commodity frequently auctioned for revenue and shared as a constrained resource.

The protection and abundant bandwidth once enjoyed by the flight test community is eroding. The scientific community is losing ground to social networks and cell phones. The new economic value of spectrum, and the politics of economic growth and voter appeasement, are the new norm.

\section{Telemetry in the Wireless Sweet Spot}

RF spectrum covers a lot of ground. It starts around $3 \mathrm{KHz}$ and ends around $300 \mathrm{GHz}$ (Fig. 1) [5]. For many services, especially broadband 
and cell phones, the unique characteristics of RF propagation and atmospheric distortion create a "sweet spot" below $3 \mathrm{GHz}$ (Fig 2) [6]. Those characteristics serve telemetry well (low signals, long distances...).

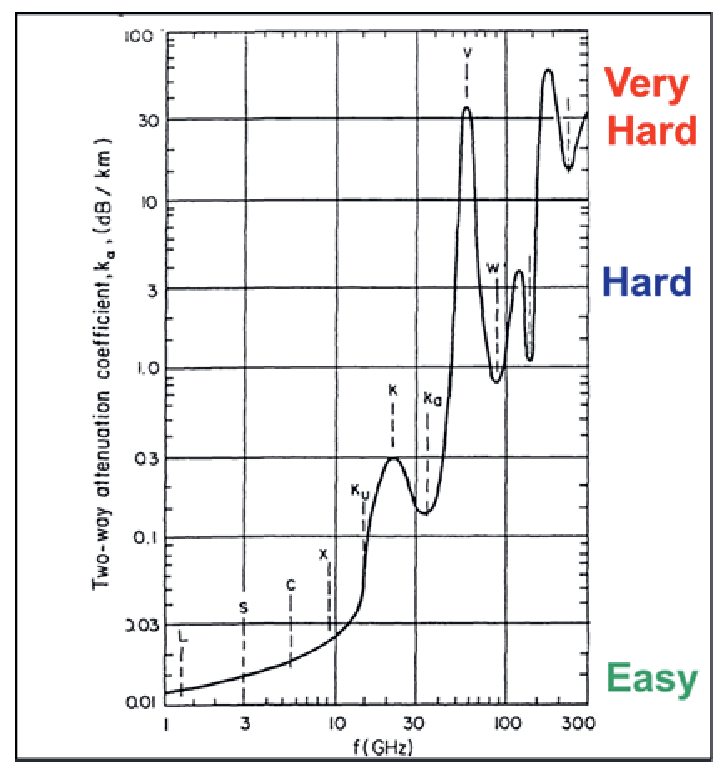

Fig. 2. Atmospheric Propagation Sweet Spot

This sweet spot also presents a unique aspect for wireless services. The best frequencies for mobile broadband are bands that: 1) can penetrate terrain and buildings (as much as possible) and 2) be received on small antennas. The range $300 \mathrm{MHz}-3.5 \mathrm{GHz}$ meets these criteria well.

This sweet spot is also the home to our AMT bands (for many of the same reasons). Many commercial services (cell phones, broadband) are aggressively seeking to displace AMT and locate in this sweet spot. It is like "waterfront" property in real estate, demanding high prices due to its short supply. The telemetry practitioner, once alone in this sweet spot, must learn to protect its property and, in some cases, involving non-safety/high-value tests, learn to share.

\section{Telemetry Economics}

The wireless service verses AMT economic comparison is difficult. A study on the economic value of telemetry in the US completed in 2006 placed a value on the $215 \mathrm{MHz}$ of available telemetry spectrum in the US at approximately $\$ 105$ billion, generating $15 \%$ of the US gross domestic product (GDP), and providing over 11 million US jobs [7]. While AMT spectrum is in the critical path for the U.S. aerospace industry. and while the data above represent a set of very strong statistics, the spectrum value when used for AMT might be challenged by its value for broadband. Several past auctions in the US has shown the virtually untapped value of spectrum as a revenue source. In 2015, the US auctioned $25 \mathrm{MHz}$ of L-Band (AWS-3 spectrum auction, known as auction 97) at a record $\$ 44.9$ billion [8]. That is far more " $\$$ per Hertz" than raised in any previous spectrum auction in the US.

\section{Surrounded by Unfriendlies}

While there are many other economic variables to consider, to the growing wireless services, to them the next step is simple: their governments should seek to auction more spectrum. AMT is surrounded by unfriendly competing services seeking our spectrum. AMT needs to survive in a congested environment.

\section{Telemetry Ninja's}

A lesson in survival techniques in unfriendly environments can be drawn from the Ninjas of ancient Japan. A Ninja was a covert agent that excelled in waging irregular warfare during the 12th-15th centuries. Ninjas figured prominently in legend and folklore, where they were associated with abilities such as invisibility and control over the natural elements. Historian Kiyoshi Watatani states that the Ninja were trained to be particularly secretive about their actions and existence [9]. Using "ninjutsu" techniques, they aim to ensure that one's opponent does not know of one's existence.

The safety issues that accompany flight test telemetry will protect our existing dedicated/primary spectrum, but the spectrum allocated for AMT is not likely to accommodate growth. Many administrations are pushing AMT to operate in shared bands. Sharing studies between AMT and other services show AMT is a difficult partner. AMT, as a small-signal service, is very sensitive to interference. Regardless, to accommodate AMT growth, we need to pursue a future that includes both dedicated and shared spectrum allocations. We must be able to survive in a shared environment, and in some cases the sharing may have to be on a secondary (noninterference) basis.

To survive outside our dedicated spectrum fortress we need to acquire new tools. Like a Ninja, the telemetry community needs to take action to survive in these commercially-driven political times. We must learn to operate in shared spectrum. We need to adopt ninjutsu techniques to ensure that our signal is not harmed by others.

To survive, telemetry ninjutsu techniques need to be developed in three main areas; DEFEND what we have, DEVELOP new ways to operate, and DISCOVER new approaches and solutions to meet our customer needs (Fig 3 ). 


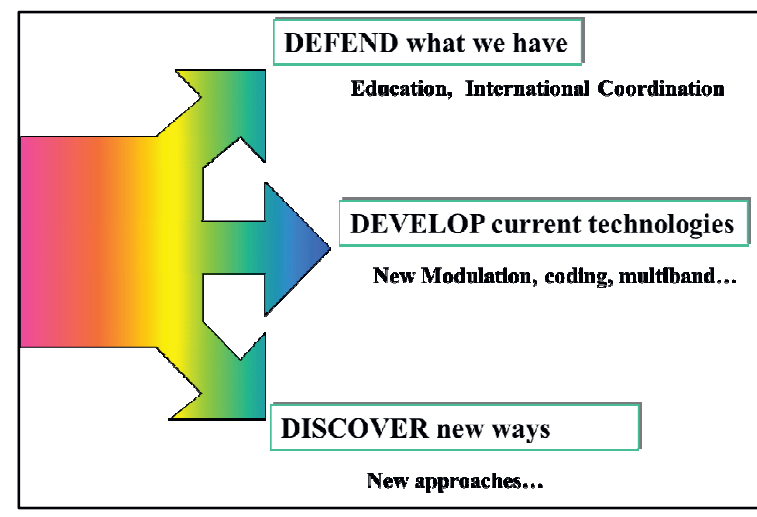

Fig. 3. 3 prongs needed for Survival

\section{DEFENDING Telemetry Spectrum}

The telemetering community needs to continually educate its decision makers. We need to tell an effective story to those in our management chains and national administrations so they have a solid appreciation for the importance of AMT spectrum. We need to make sure they have a good understanding and current information so they can make informed decisions and inputs.

\section{Calling All Ninjas}

To aid in "getting the message out" the International Consortium for Telemetry Spectrum (ICTS), chartered by the International Foundation for Telemetering, was created to "establish an international information exchange of telemetry practitioners to promote the benefits and enhancement of electromagnetic spectrum for telemetering applications" [10]. The main goal of the ICTS is to prepare the international telemetering community to address international concerns and threats presented at the World Radiocommunication Conferences (WRCs), the forum chartered by the ITU. The ICTS outreach effort was very effective in supporting the campaign to secure additional telemetry spectrum that resulted in the new C-Band telemetry band we recognize today at WRC-07 [11].

The ICTS is able to help defend AMT spectrum by working within the grass-roots scientific telemetry community. The ICTS needs to effectively engage the affected industries, manufacturers, academia, and government agencies that work with, and rely on, telemetry. Organizations like the ICTS needs to expand as a resource library that will help the telemetry community stress their needs to their management and national administrations, and eventually, the WRC.

\section{DEVELOPING Current Technology}

The telemetering community cannot rest on their current technology or spectrum. In the past we have relied on high walls (primary assignments) and few enemies (very little commercial wireless) for our protection. Now we must learn to live closer to the enemy's camp (other users). The flight test telemetry community has been working in this area for a long time. In the US, the Test Resource Management Center (TRMC) has funded several major projects (Advanced Range Telemetry (ARTM) [12], Integrated Network Enhanced Telemetry [iNET] [13]) and research efforts (Spectral Efficient Technologies) [14] that have paid off many times over in efficiencies and agility increases. Much more is needed.

\section{Ninja Efficiency; Doing More with Less}

While the telemetry need has grown (Fig 4) the US has been able to avoid significant cancellations and impacts by implementing these new technologies developed by several research programs. Even with the available telemetry spectrum shrinking by $35 \%$ (previous to WRC 07) they have accommodated an exponential data growth by increasing the $\mathrm{bits} / \mathrm{Hz}$ efficiency of RF telemetry. The implementation of higher order modulations (like Shaped Offset Quadture Phase Shift Keying (SOQPSK) and Continoues Phase Modulation (CPM), via the ARTM program) has

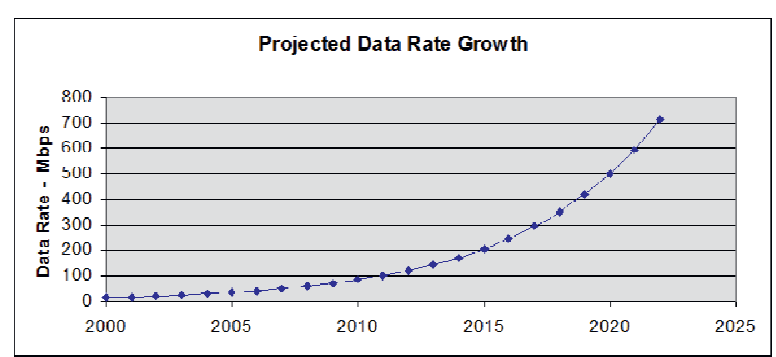

Fig. 4. Projected Telemetry growth in the US

resulted in many users achieving a 200\% increase in data effeciency [15]. Without this and other improvements in quality of service, there would have been serious consequences to telemetry users. The US has achieved a major cost avoidance by the implementation of these new telemetry technologies. These efficiencies can be seen in the Yuma, California tests of an error-free AMT link on a helicopter recently accomplished by the Army [16]. The cost of delays, aborts, or cancels in a test program can be significant. One delayed or canceled test mission due to the unavailability of telemetry spectrum could cost a US program manager in excess of $\$ 1$ million [17].

We need to develop more techniques, like higher order modulation, to make telemetry more efficient, to be able to transmit more data with less spectrum. 


\section{Ninja Flexibility; Adapting and Adjusting}

Another ninjutsu skill -- the "telemetrist" (a user, supplier, or customer of telemetry) needs to develop is the Ninja's ability to improve their use of existing weapons in their arsenal. Ninja's were known for developing new techniques to increase the agility of their current weaponry in new and different ways.

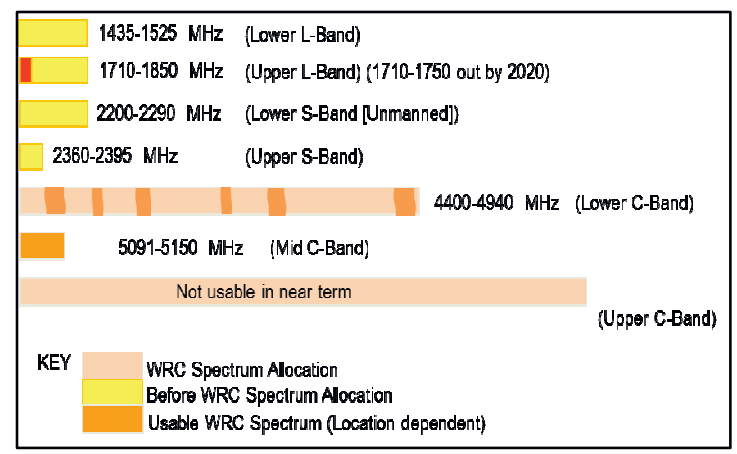

Fig. 5. RF Telemetry Bands Available (ITU Region 2 Shown)

One new weapon is telemetry frequency agility. We need the agility to access all spectrum bands available for telemetry. At WRC-07 we gained access to several new bands (Fig 5). To exploit these widely scattered telemetry bands we need to build more frequency agile technology. The development of multiband transmitters, antennas, cabling, and associated components is critical for exploiting these bands. The ability to tune, in real-time, to multiple bands will not only allow us to schedule more missions, but to avoid conflicts before they affect our mission. Additional research into software-defined radios and wide-band components is critical to achieve this desired telemetry frequency agility.

\section{Telemetry Honor; Sharing and Respect}

The sweet spot spectrum is congested. In areas where there are limited AMT authorizations (like Asia and the Pacific), AMT spectrum use for non-safety of life/non-high value purposes might be on a secondary basis. The telemetrist must give preference in such cases to the primary service by preventing interference. With Ninja-like honor, we need to accept interference from others, and not interfere with them.

Telemetry is by its low spectral power density nature an interference-limited technology. Our test articles typically have significant size, weight, and power limitations that restrict our transmission options. As a "small-signalservice", we are sensitive to interference even from low-power components (such as cell phones). A cell phone near a telemetry dish will over-power reception from a test article telemetry signal when the antenna is 100 miles away or even less.

Recent research into Low Probability of Detect Codes (LPDC), Space-Time-Coding (STC), error-correction, and other solutions can increase our noise tolerance and preserve our quality of service (QoS). As seen in several papers presented at telemetry conferences and other venues (European Test and Telemetry Conference, International Telemetering Conference [18], International Test and Evaluation Associations [ITEA] Test Instrumentation Workshop [19]) these technologies are maturing rapidly and many are now available commercially.

\section{Risk Aversion as a Barrier}

Telemetry executives need to manage risk better and become less new-technology averse. Once a technology is proven and reliable, they typically do not want to change it. Their job is not to test telemetry systems, but to use them to test something else. Telemetry is a critical tool that needs to be sufficiently mature (robust and reliable) so it can be relied on to do the real test job (air vehicles, rockets, and other aeronautical product development). Telemetry systems need to be low-risk to do a high-risk mission. This has caused many to be too comfortable with old style telemetry such as, pulse code modulation/frequency modulation (PCM/FM), and current systems. We need to get comfortable with a new slew of acronyms (SOQPSK, CPM, LPDC, STC...).

The best way to do this is plan to implement them earlier than when you may need them. As a sailor, my Dad told me the "best time to reef the main is before you need it" [20]. This can also be true of new technology. Ranges and Test Facilities need to implement frequency agile, spectral efficient and robust technologies before the spectrum congestion demands it. e know our future will be more spectrally congested and sharing of frequency bands will become the new norm. Range and facility managers who rely on telemetry today need to make proactive investments in new technology to reserve the utility of their telemetry tools in the future.

\section{DISCOVERING New Telemetry Frontiers}

"... and now for something completely different..."

-Monty Python

The modern Ninja does not look or act like his 12th century relative. Today's Ninjas are called Special Ops, Rangers, or many other terms that 
denote the progression and development of the ninjutsu skill-set. They have been constantly redefined and reinvented. Telemetry needs to be "reinvented" also.

As cell phone technology can be categorized into multiple generations, the telemetry community is rapidly approaching a similar evolutionary path. As our 4G smart phones bear little resemblance to the $2 G$ flip phones of just 10 years before, tomorrow's telemetry system will diverge also. This can be visualized as "Telemetry Generations" (Fig 6).

- 1st Generation - frequency modulation (1960's)

- 2nd Generation -digitally encoding (1970's)

- 3rd Generation -advanced modulation (2000's)

- 4th Generation - Network-packets (2020's)

- 5th Generation - ??

Fig. 6. Telemetry Generations

In figure 6 each generation had a significant change in architecture from the previous. Each generation has implementation challenges but can achieve increased capabilities as a result. In some cases, generational leaps can be caused by "disruptive technology". A disruptive technology is a technical innovation that eventually displaces an existing technology. Not all technical innovations are disruptive, even if they are revolutionary. Telephone technology was a disruptive technology that eventually replaced the telegraph for long distance communication. In telemetry, we need more of these "disruptive technology" to move forward.

Ranges typically have a hard time adopting new technologies due to a legacy sensitivity that can slow the introduction of distributive technology. This is due impart to the sizable capital investment in test article instrumentation and range infrastructure. This investment cannot be easily ignored and discarded (as is done with cell phones). As a result, Ranges will typically overlap technologies via downward compatibility. The Ranges need for compatibility with legacy systems constrain the introduction of beneficial disruptive technology. On some of the largest Ranges, the period for these generational leaps has been measured in decades.

Some examples of what could be that disruptive technology that drives $5 \mathrm{G}$ telemetry may be new bands and commercial technology.

\section{New Bands; Ninja Climbing Skills}

Can telemetry survive outside the less-than 3 $\mathrm{GHz}$ "sweet spot"? Sometimes to avoid conflict with an enemy, a Ninja needs to climb up to freedom.
Aggressive research should continue to enable telemetry systems in higher spectral bands (one way to avoid the high cost of waterfront property is to move to the desert). The higher $\mathrm{RF}$ bands ( $\mathrm{X}, \mathrm{Ku}, \mathrm{K}, \mathrm{KA}, \mathrm{V}, \mathrm{W})$ present many atmospheric and physical challenges that need aggressive research and innovation. Managers of test ranges and facilities should be promoting and leading this research.

\section{New Technology; Ninja Weaponry Advances}

As a Ninja adopts new weapons, the innovative telemetrist needs to look beyond the current telemetry paradigm. In the early years of telemetry, the telemetrist was a driving force in wireless communication. Today we are a small niche market for a handful of (awesome) companies. The telemetry stakeholders (suppliers and customers) need to promote research into new technologies and concepts that can operate in a shared spectral environment.

One of these disruptive technologies to telemetry maybe Long-Term Evolution (LTE) [21]. Joining the $4 G$ cell phone industry maybe a future step for flight test telemetry. Research is ongoing in the US and several other countries on the utilization of LTE and $4 G$ technology to take the next generational leap for telemetry. Using this concept of cells and LTE networks to collect and distribute real-time data from fast vehicles presents many interesting challenges (Doppler shifts, multipath, handoffs, QoS, security,...) that should be studied by the Ranges and Test Facilities.

\section{Better Ninja Stealth}

Taking the Ninja allegory (maybe too far?), what if telemetry went completely stealth? Additional research and testing of stealth telemetry technologies (directional antennas spread-spectrum, burst, LASER ...) could preclude the need for telemetrist to be seen (and schedule) with others. As the potential for interference is reduced, we may achieve complete Ninja stealth, the ability to operate in any band at any time, no need to schedule or manage outside the telemetry community.

Going "directional" shows potential telemetry promise. One of the nice features of many of our test articles is we know where they are (typically we have good time-space positioning information). If we know the test article position, and the ground antenna position, could we derive a predicted pointing solution for antenna tracking? Research outside the omnidirectional antenna model can have significant payback. Also, with a directional beam, power draw is reduced, interference reduced, and scheduling can be simplified. Research into directional antennas, phased-array applications, and 
lobe/null tuning is showing great promise and needs to be matured.

Several other "stealth" techniques have been explored and should be developed. Pseudorandom noise-based spread spectrum signals allow the telemetry signal to hide in the noise floor. Systems that collect the data on-board and burst the signal over a high-capacity (microwave/LASER) link periodically needs further investigation. This application of "we can see them, but they can't see us" telemetry technology should be pushed and developed.

\section{The Need for Innovation}

The Range and test facility management needs to respond to the congested spectral environment pro-actively. Telemetry is in need of massive influxes of innovation. Some of these innovations presented in this paper are under development. The threat (spectrum congestion, spectrum encroachment, spectrum sharing) is real and will grow. Like a Ninja, we need to adapt to the enemy at hand.

We cannot expect change if we always do what we have always done (that is the definition of insanity).

\section{AMT FUTURE; A call for Super-Ninja Action}

The international telemetering community is facing very aggressive threats that will shape a new Super-Ninja paradigm.

AMT users have found themselves with less spectrum in which to operate.

This community has a critical need for better stealth telemetry tools and methodologies to accommodate this new "unfriendly skies" paradigm. When we must operate in bands where we lack a primary allocation, we must develop Ninja-like skills.

Our safety aspects will always require interference protection, as will other flight tests which, while not necessarily involving safety-offlight, may entail other high value factors. This protection is restricted to a few AMT bands. The new paradigm presented in this paper is that of secondary AMT uses (non-safety-offlight/non-high-value tests) in shared bandssomething new to the telemetry community. This could present itself as a significant spectrum model in the future.

In this future, we must function with the stealth of a Ninja to avoid causing interference while in turn avoiding interference from primary users in the band. We must share with honor and respect in this new age. The Range and test facility managers need to aggressively seek new research and develop new "super Ninja" technology to remain effective in congested shared spectral environments. Without their direct involvement, the AMT community will be unprepared to react to the new paradigm.

\section{References}

[1] [1] "Those Were the Days" song lyrics by Gene Raskin, who put English lyrics to the Russian song "Dorogoi dlinnoyu" ("дорогой длинною"), written by Boris Fomin (1900-1948) with words by the poet Konstantin Podrevskii.

[2] History of Telemetry, http://www.orielsystems.com/short-historytelemetry/ (as of 3/20/2018)

[3] Aerospace and Flight Test Radio Coordinating Council , https://aftrcc.org/

[4] Exploring the Value and Economic Valuation of Spectrum, John Alden, ITU Broadband Series Report, 2012 http://www.itu.int/ITUD/ict/material/FactsFigures2010.pdf .

[5] http://www.esa.int/var/esa/storage/images/esa_m ultimedia/images/2013/11/satellite_frequencies (as of $3 / 20 / 2018$ )

[6] The Great Radio Spectrum Famine, Institute of Electrical and Electronics Engineers Spectrum Magazine, http://spectrum.ieee.org/telecom/wireless/thegreat-radio-spectrum-famine

[7] Economic Impact of Telemetry and Its Essential Role in the Aerospace Industry, Darrell E. Ernst and Carolyn A. Kahn, MITRE TECHNICAL REPORT Project No.0703D110-AA, 2006

[8] IT'S OVER: FCC's AWS-3 spectrum auction ends at record \$44.9B in bids, Phil Goldstein, https://www. fiercewireless.com/wireless/it-s-overfcc-s-aws-3-spectrum-auction-ends-at-record-449b-bids

[9] [9] From Wikipedia, "Ninja" https://en.wikipedia.org/wiki/Ninja (as of 3/23/2018)

[10] International Consortium for Telemetry Spectrum. http://www.telemetryspectrum.org

[11] Resolutions 416 and 418 in the FINAL ACTS of the World Radiocommunication Conference (WRC-2007), Radiocommunications Sector, International Telecommunications Union, , Geneva, 2007

[12] The Advanced Range Telemetry (ARTM) program is described in RANGE TELEMETRY IMPROVEMENT AND MODERNIZATION, Chalfant, Timothy A.; Irving, Charles :Edwards Air Force Base, Proceedings from the International Telemetering Conference, 1997

[13] The integrated Network Enhanced Telemetry (iNET) program is described in INTEGRATED NETWORK-ENHANCED TELEMETRY, Skelley, Dan S., Naval Air Warfare Center, Issue Proceedings from the International Telemetering Conference. 
[14] The Spectrally Efficient Telemetry program is under the Test and Evaluation Science and Technology effort managed by the Test Resource Management Center (DOD AT\&L), US Department of Defense.

[15] Description and Performance Results for the Advanced Range Telemetry (ARTM) Tier II Waveform, M. Geoghegan, Proceedings of the International Telemetering Conference, 2000

[16] ROTARY-WING FLIGHT TESTS TO DETERMINE THE BENEFITS OF FREQUENCY AND SPATIAL DIVERSITY AT THE YUMA PROVING GROUND, Diehl, Michael; Swain, Jason; Wilcox, Tab, US Army Air Combat Systems Directorate, Proceedings from the International Telemetering Conference, 2016
[17] Economic Impact of Telemetry and Its Essential Role in the Aerospace Industry, Darrell E. Ernst and Carolyn A. Kahn, MITRE TECHNICAL REPORT Project No.0703D10-AA, 2006

[18] International Foundation for Telemetering www.telemetry.org

[19] International Test and Evaluation Association. www.itea.org

[20] Attributed to George A. Chalfant MD (19242012), of the sailing vessel WindSwift

[21] Long Term Evolution, in 3 GPPP standard for cell phones 